\title{
Identifying Challenges to Quality in Preconception Health Care among Women of Reproductive Age in Lipa City, Batangas
}

\author{
Carmencita D. Padilla, $, 1,2,3,4$ Aster D. Lynn Sur, ${ }^{2}$ Katrina D.Villarante, ${ }^{5}$ Howell D. Crisostomo, ${ }^{\dagger 2}$ \\ Ariel G. Lescano, ${ }^{6}$ Patrick Jose D. Padilla, ${ }^{2,7}$ Rufus Thomas Adducul, ${ }^{2}$ Lita L. Orbillo, ${ }^{8}$ Anthony P. Calibo, ${ }^{8}$ \\ Juanita A. Basilio, ${ }^{3}$ Salimah R. Walani ${ }^{4,9}$ and Christopher P. Howson ${ }^{4,10}$ \\ ${ }^{1}$ Department of Pediatrics, College of Medicine and Philippine General Hospital, University of the Philippines Manila \\ ${ }^{2}$ Institute of Human Genetics, National Institutes of Health, University of the Philippines Manila \\ ${ }^{3}$ Newborn Screening Reference Center, National Institutes of Health, University of the Philippines Manila \\ ${ }^{4}$ March of Dimes Global Network for Maternal and Infant Health \\ ${ }^{5}$ Department of Family and Community Medicine, College of Medicine and Philippine General Hospital, University of the Philippines Manila \\ ${ }^{6}$ Lipa City Health Office, Philippines \\ Department of Obstetrics and Gynecology, College of Medicine and Philippine General Hospital, University of the Philippines Manila \\ ${ }^{8}$ Department of Health, Manila, Philippines \\ ${ }^{9}$ March of Dimes, Arlington, Virginia, USA \\ ${ }^{10}$ Howson $E$ Partners for Global Health, Santa Fe, New Mexico
}

\begin{abstract}
Background. Preconception care is a set of interventions that aim to identify and modify biomedical, behavioral, and social risks to a woman's health or pregnancy outcome through prevention and management. These interventions emphasize factors that must be addressed before conception or early in pregnancy in order to have maximal impact. Preconception health care is a key intervention in improving maternal and neonatal health care. Identification of specific needs of population-at-risk remains crucial in developing quality preconception health care programs in the Philippines.

Objectives. This paper: 1) described the preconception health status of women of reproductive age in selected communities in Lipa City Batangas; 2) identified the perceived preconception needs of women of reproductive age in selected communities; 3) determined the significant challenges to the provision of appropriate preconception health care; and 4) provided recommendations to address the gaps and challenges.
\end{abstract}

Methods. A total of 4,357 women of reproductive age were interviewed using a preconception checklist tool previously developed by researchers from Peking University (China), American University of Beirut (Lebanon), and University of the Philippines Manila (Philippines). Eleven Focus Group Discussions (FGDs) on various aspects of preconception health care were conducted among women of reproductive age from communities and workplaces (industries/factories, government offices, schools, entertainment centers, health centers). Thematic analyses of the data from the FGDs were performed. Recommendations for overcoming identified challenges to quality services were presented.

Results. There are salient gaps in preconception health care, particularly in micronutrient intake, immunization status, family planning and infectious diseases screening in both urban and rural communities. The study also showed major gaps in medical and educational services, particularly for adolescents.

Paper presented at the International Conference on Birth Defects and Disabilities in the Developing World, November 2013, Cebu, Philippines.

Corresponding author: Carmencita D. Padilla, MD, MAHPS Institute of Human Genetics National Institutes of Health

University of the Philippines Manila

Pedro Gil St., Ermita, Manila 1000, Philippines

Email: cdpadilla@up.edu.ph
Conclusion. Health and social challenges in the preconception health care delivery system for women of reproductive age in Lipa City Batangas were identified, including the prioritization of at-risk groups and development of strategies to address preconception health care gaps in both urban and rural settings. The alarming increasing rate of teenage pregnancy must be given highest priority with integration of safe and 
healthy pregnancy in the curriculum. The development of programs for men and women recognizes that parenthood is a partnership. To guarantee a successful program on preconception health care services, government must utilize an inter-sectoral and interdisciplinary approach with the participation of various stakeholders and sectors, both government and private. The engagement of women of reproductive age in planning provides a dynamic feedback for the relevance of the planned programs.

Key Words: Preconception health care, preconception checklist, workplace wellness modules

\section{INTRODUCTION}

The Centers for Disease Control and Prevention (CDC) has defined preconception health as "... the health of women and men during their reproductive years. Preconception health focuses on steps that women, men and health professionals can take to reduce risks, promote healthy lifestyles, and increase readiness for pregnancy. Although preconception health care emphasizes preparing for pregnancy, all women and men of reproductive age can benefit, whether or not they plan to have a baby one day. ${ }^{\text {"1-3 }}$ Indeed, preconception health care is a key intervention in improving maternal and neonatal health care. Identifying the specific needs of at-risk population is crucial in developing preconception health care programs. ${ }^{3}$

Preconception care or preconception health carerelated programs exist in different forms around the world. With universal interest in attaining Millennium Development Goals (MDGs) and Sustainable Development Goals (SDGs), the preconception period has emerged as a focus for continued improvement of maternal and child health. ${ }^{4,5}$ For this study, preconception care was defined as a set of prevention and management interventions aimed at identifying and modifying biomedical, behavioral, and social risks to a woman's health or pregnancy outcome. These interventions emphasize items requiring action before conception or early in pregnancy in order to have maximum impact. Preconception care begins before the first pregnancy and continues through any subsequent pregnancies. It targets all women and couples of childbearing age and is not limited to those planning a family since a significant proportion of pregnancies are unplanned.

Many countries have recognized the importance of preconception care and thus, established comprehensive health policies. In Hong Kong, a pre-pregnancy preparation service provides risk assessment, an educational video on pregnancy preparation, a physical exam, and laboratory tests. Women are advised to take folic acid, undergo screening for sexually transmitted infections and thalassemia, and have vaccinations for hepatitis B and rubella. ${ }^{6}$ In China, a national policy, made non-compulsory in 2003, previously required couples to undergo a premarital medical exam that included a history of hereditary and mental problems, a physical exam, and laboratory tests. Reported benefits included diagnosis of HIV, prevention of transmission of hepatitis B and reduced rates of neural tube defects. ${ }^{6-8}$ In South Korea, as a result of a decreased birth rate, increased adverse pregnancy outcomes, and low folic acid intake, a preconception care program was established that included education both for doctors and for women of reproductive age, and clinic services. The Preconception Counseling Clinic provides a physical examination, laboratory tests, and screening for risk factors. ${ }^{6}$ In the Philippines, there is currently no national comprehensive policy on preconception health care. Department of Health (DOH) efforts include programs focused on nutrition of women of reproductive age (nutrition counseling, iron and folic acid supplements), family planning, adolescent health services, prevention and treatment of lifestyle diseases and infections, and deworming. ${ }^{9}, 10,11$

The objectives of the paper were: 1) to describe the preconception health status of women of reproductive age in selected communities in Lipa City, Batangas; 2) to identify the perceived preconception needs of women of reproductive age; 3 ) to determine the significant challenges to the provision of appropriate preconception health care; and 4) to provide recommendations to address the gaps and challenges.

\section{METHODS}

\section{Study site}

Lipa City was selected as a study site because of its progressive local health programs. Located $86 \mathrm{~km}$ South of Manila, it has 72 barangays with 12 urban barangays in the city proper, 12 'emerging urban' barangays and 48 rural barangays. The projected number of females in Lipa City was 143,269 at the time of the study. ${ }^{12}$ Lipa City Batangas, through its City Health Officer (CHO), was invited to participate in the study. The $\mathrm{CHO}$ agreed and identified the barangays for inclusion in the study.

\section{Preconception health assessment and screening tool}

The project used a preconception health assessment and screening clinical tool developed and implemented previously by three (3) collaborating centers in the March of Dimes Foundation's Global Programs and Global Network for Maternal and Infant Health (GNMIH) - Peking University (China), American University of Beirut (Lebanon), and University of the Philippines (UP) Manila (Philippines). ${ }^{3}$ The tool included questions on family planning, reproductive health history, genetic risks, immunizations, micronutrients, lifestyle, infectious diseases, chronic medical conditions, psychosocial risk factors and socioeconomic conditions. This tool served as a guide during the interview of women of reproductive age (15-49 years) from selected areas of Lipa City. Interview responses were 
written directly on the preconception checklist tool by the interviewer. From September 2011 to December 2012, 4,357 women were interviewed. From August 2012 to January 2013, eleven FGDs were organized among selected study participants. FGDs were facilitated by the study team consisting of trained nurses and physicians. Thematic analyses were performed using the FGD transcripts. Direct Tagalog to English translations were done by the research assistants for the benefit of non-Filipino readers.

\section{Ethics review}

The study was granted approval by the Ethics Review Board of the National Institutes of Health, UP Manila. Informed consent was obtained from all participants prior to the interviews and the FGDs.

\section{RESULTS}

\section{Interviews}

A total of 4,357 women of reproductive age were interviewed from September 2011 to December 2012. Of the 4,357 women, 1,459 were from various workplaces and 2,898 were from the community settings. To maximize recruitment of study participants of women of reproductive age, interviews were conducted in various settings which included workplaces and community settings.

Project orientation and collaboration were conducted among local government officers at the city and community level and among the key people of the various workplaces.

House to house interviews were conducted in the target communities with the assistance of the community leaders. For workplaces, interviews were facilitated by their respective Human Resource Office allowing their workers or employees to be interviewed during workhours.

Table 1 presents the breakdown of participants according to study sites. Table 2 shows the breakdown of participants according to age. Half (50.98\%) of the women completed secondary school education, $39.2 \%$ started but did not complete secondary school education and $9.96 \%$ completed primary school education. Table 3 presents the summary of responses using the preconception checklist tool.

Table 1. Distribution of participants according to study sites

\begin{tabular}{lrc}
\multicolumn{1}{c}{ Study Site } & No. of Participants \\
Workplaces & 310 & 1459 \\
Industries/factories & 84 & \\
City Hall/local government offices & 24 & \\
Other offices & 968 & \\
School (6 teachers \& 962 students) & 14 & \\
Entertainment Centers & 59 & \\
Health Centers & & 2898 \\
Community Settings & 1,926 & \\
$\quad$ Rural & 693 & \\
Urban & 279 & \\
$\quad$ Pre-marriage counseling Seminars & & 4357 \\
\hline Total &
\end{tabular}

\section{Family planning and reproductive spacing}

Of the 4,357 women, 657 (15.08\%) used family planning methods. Table 4 presents the utilization of family planning methods according to age group. Table 5 presents the frequency of the different modes among the 657 users and in relation to the total number of participants.

Table 2. Distribution of patients according to age

\begin{tabular}{cc} 
Age & No. of Participants \\
$15-19$ & 954 \\
$20-24$ & 1,009 \\
$25-29$ & 783 \\
$30-34$ & 631 \\
$35-39$ & 473 \\
$40-44$ & 355 \\
$45-49$ & 152 \\
\hline Total & 4,357 \\
\hline
\end{tabular}

Table 3. Summary of responses from Women of Reproductive Age in Lipa City

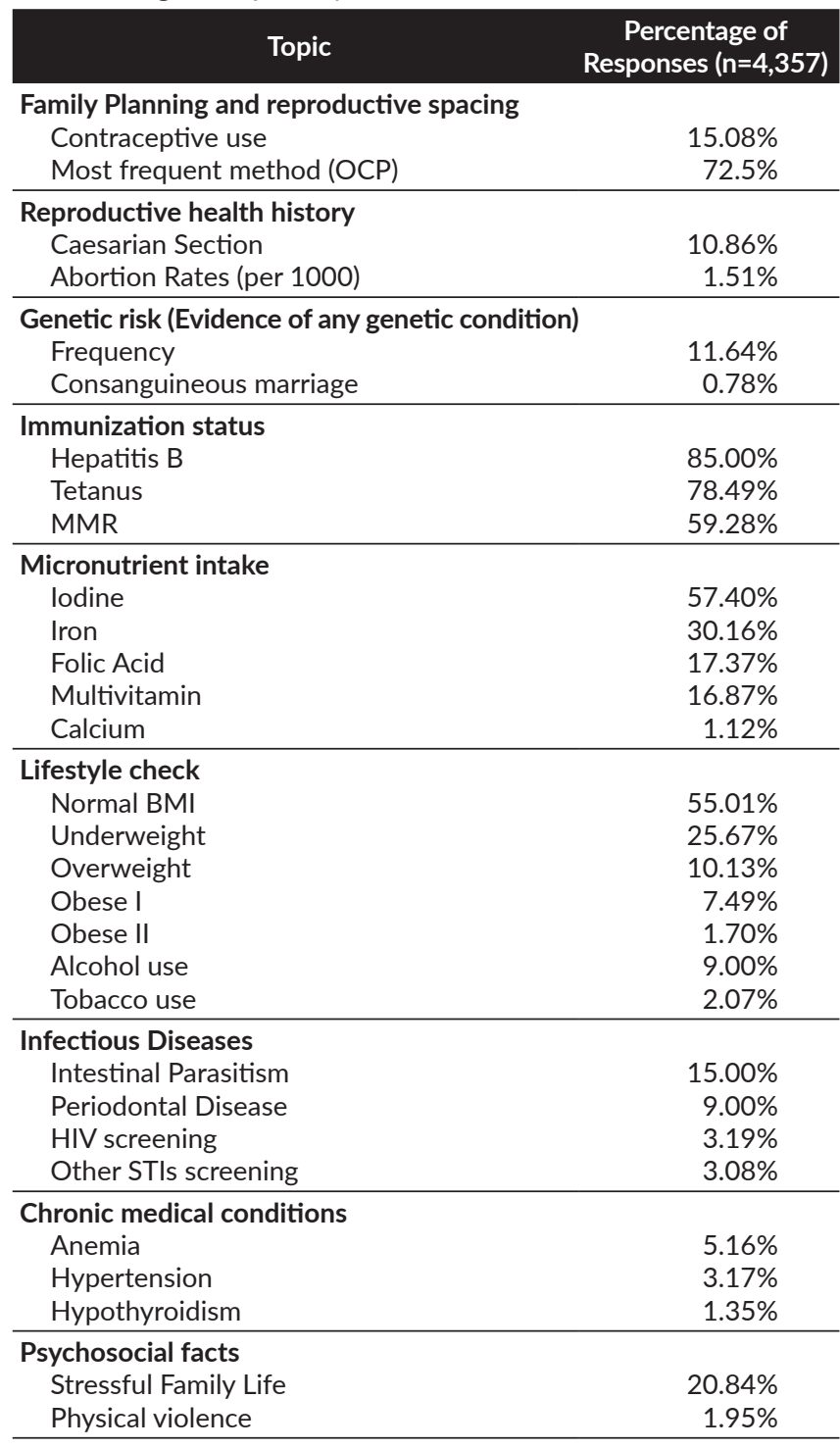

OCP - Oral Contraceptive Pills 
Table 4. Utilization of family planning methods according to age group and workplace $(n=657)$

\begin{tabular}{|c|c|c|c|c|c|c|c|c|c|c|}
\hline \multirow[t]{2}{*}{ Age } & \multicolumn{2}{|c|}{$\begin{array}{l}\text { Women in industries } \\
\qquad(n=310)\end{array}$} & \multicolumn{2}{|c|}{$\begin{array}{l}\text { Local Government } \\
\qquad(n=84)\end{array}$} & \multicolumn{2}{|c|}{$\begin{array}{l}\text { Other offices } \\
(n=24)\end{array}$} & \multicolumn{2}{|c|}{$\begin{array}{l}\text { Teachers } \\
(\mathrm{n}=6)\end{array}$} & \multicolumn{2}{|c|}{$\begin{array}{l}\text { Entertainers } \\
\quad(n=14)\end{array}$} \\
\hline & $\mathbf{N}$ & $\%$ & $\mathbf{N}$ & $\%$ & $\mathbf{N}$ & $\%$ & $\mathbf{N}$ & $\%$ & $\mathbf{N}$ & $\%$ \\
\hline $15-19$ & 3 & 0.97 & - & - & - & - & - & - & - & - \\
\hline $20-24$ & 9 & 2.90 & 1 & 1.19 & 2 & 8.33 & - & - & 3 & 21.43 \\
\hline $25-29$ & 11 & 3.55 & 2 & 2.38 & 1 & 4.17 & 1 & 16.67 & 2 & 14.29 \\
\hline $30-34$ & 9 & 2.90 & 4 & 4.76 & 2 & 8.33 & - & - & 1 & 7.14 \\
\hline $35-39$ & 6 & 1.94 & 5 & 5.95 & - & - & - & - & - & - \\
\hline $40-44$ & 4 & 1.29 & 7 & 8.33 & - & - & - & - & - & - \\
\hline $45-49$ & 2 & 0.65 & 2 & 2.38 & - & - & - & - & - & - \\
\hline Total using FPM & 44 & 14.19 & 21 & 25.00 & 5 & 20.83 & 1 & 16.67 & 6 & 42.86 \\
\hline
\end{tabular}

$n$ - number of respondents FPM - family planning method

Table 5. Frequency of the different modes of family planning methods among the users $(n=657)$ and the total number of participants $(n=4,357)$

\begin{tabular}{lrrc}
$\begin{array}{c}\text { Modes of Family } \\
\text { Planning Methods }\end{array}$ & Number & \% of users & $\begin{array}{c}\text { \% of total } \\
\text { participants }\end{array}$ \\
\hline Oral Contraceptive Pills & 474 & 72.15 & 10.88 \\
Condoms & 76 & 11.57 & 1.74 \\
Tubal Ligation & 42 & 6.39 & 0.96 \\
Injectables & 34 & 5.18 & 0.78 \\
IUD & 13 & 1.98 & 0.30 \\
Withdrawal & 10 & 1.52 & 0.23 \\
Calendar & 7 & 1.07 & 0.16 \\
Others & 1 & 0.15 & 0.02 \\
\hline Total & 657 & 100.00 & 15.08 \\
\hline
\end{tabular}

\section{Number of children and manner of delivery and abortion rates}

The mean number of children was 1.61 compared to 3.0 children per woman in the 2013 National Demographic and Health Survey. ${ }^{13}$ Delivery via cesarean section was $10.86 \%$ lower than the national data of $22 \% .{ }^{14}$ The abortion rate was also lower at 15.1 per 1,000 compared to the national rate of $22-31$ per $1,000 .{ }^{15}$

\section{Reproductive health history}

Of the 1180 women with at least 1 successful pregnancy, 473 (40\%) delivered by Cesarean Section. Problematic outcomes were noted in 707 (16.23\%) pregnancies, i.e. preterm birth, low birth weight, neonatal death, infant death, stillbirth, or a birth defect. (Table 6)

\section{Genetic Risk}

Table 7 shows that $31.26 \%$ (1,362 of 4,537) reported that she, her spouse or siblings had a risk for a perceived genetic condition. It is important that mothers have knowledge of adverse pregnancy outcomes (recurrent miscarriages, pre-terms, stillbirths, low birth weight) and this information can be included in preconception care programs designed to lessen the chances of recurrence. Screening for a family history of genetic conditions is also a
Table 6. Frequency of problematic outcomes among women with at least 1 pregnancy $(n=707)$

\begin{tabular}{lccc}
$\begin{array}{c}\text { Reproductive } \\
\text { Outcome }\end{array}$ & Number & $\begin{array}{c}\text { \% of women with at } \\
\text { least 1 pregnancy }\end{array}$ & $\begin{array}{c}\text { \% of total } \\
\text { participants }\end{array}$ \\
\hline Preterm birth & 99 & 8.39 & 2.27 \\
Low Birth Weight & 72 & 6.10 & 1.65 \\
Neonatal Death & 52 & 4.41 & 1.19 \\
Infant death & 34 & 2.88 & 0.78 \\
Miscarriage & 353 & 29.92 & 8.10 \\
Stillbirth & 32 & 2.71 & 0.73 \\
Birth defect & 65 & 5.51 & 1.49 \\
\hline Total & 707 & 100.00 & 16.23 \\
\hline
\end{tabular}

Table 7. Percentage of women with relatives with genetic conditions ( $n=1,362$ )

\begin{tabular}{lccc} 
With Genetic Condition & Numbers & \% of the 1362 & \% of 4357 \\
\hline Women Respondents & 507 & 37.22 & 11.64 \\
Siblings & 458 & 33.63 & 10.51 \\
Spouse & 255 & 18.72 & 5.85 \\
Spouse Siblings & 142 & 10.43 & 3.26 \\
\hline Total & 1,362 & 100.00 & 31.26 \\
\hline
\end{tabular}

component of preconception care and identification of such risks allows for genetic counseling, reproductive planning, and early intervention whenever possible., ${ }^{2,16}$

\section{Immunization}

Although not $100 \%$ uptake, there is an active immunization program. Table $8 \mathrm{a}$ presents the rates of the immunization coverage. Table $8 \mathrm{~b}$ presents the immunization status by age. Table $8 \mathrm{c}$ presents the immunization status by study site. The 3 vaccines reduce the risk for congenital rubella syndrome, vertical transmission of hepatitis $B$, and neonatal tetanus. ${ }^{2,17}$

\section{Micronutrient Intake}

Overall data on micronutrient intake showed low utilization of micronutrients. Table $9 \mathrm{a}$ presents frequency of micronutrient intake according to age and Table $9 \mathrm{~b}$ presents frequency according to study site. Other countries, 


\begin{tabular}{|c|c|c|c|c|c|c|c|c|c|c|c|}
\hline \multicolumn{2}{|c|}{$\begin{array}{l}\text { Health workers } \\
\qquad(\mathrm{n}=59)\end{array}$} & \multicolumn{2}{|c|}{$\begin{array}{l}\text { Urban community } \\
\text { women }(n=693)\end{array}$} & \multicolumn{2}{|c|}{$\begin{array}{l}\text { Rural community } \\
\text { women ( } n=1926)\end{array}$} & \multicolumn{2}{|c|}{$\begin{array}{l}\text { School } \\
(n=962)\end{array}$} & \multicolumn{2}{|c|}{$\begin{array}{c}\text { Pre-marriage } \\
\text { seminar }(n=279)\end{array}$} & \multicolumn{2}{|c|}{$\begin{array}{l}\text { Total No. of FP Users } \\
\text { according to age } \\
(n=657)\end{array}$} \\
\hline$N$ & $\%$ & $\mathbf{N}$ & $\%$ & $\mathrm{~N}$ & $\%$ & $N$ & $\%$ & $\mathbf{N}$ & $\%$ & $\mathbf{N}$ & $\%$ \\
\hline- & - & 3 & 0.43 & 11 & 0.57 & 5 & 0.52 & - & - & 22 & 3.35 \\
\hline- & - & 21 & 3.03 & 55 & 2.86 & 11 & 1.14 & 6 & 2.15 & 108 & 16.44 \\
\hline- & - & 28 & 4.04 & 106 & 5.50 & 8 & 0.83 & 3 & 1.08 & 162 & 24.66 \\
\hline 3 & 5.08 & 35 & 5.05 & 93 & 4.83 & 3 & 0.31 & 7 & 2.51 & 157 & 23.90 \\
\hline 4 & 6.78 & 26 & 3.75 & 75 & 3.89 & - & - & - & - & 116 & 17.66 \\
\hline 6 & 10.17 & 15 & 2.16 & 42 & 2.18 & - & - & - & - & 74 & 11.26 \\
\hline 1 & 1.69 & 4 & 0.58 & 9 & 0.47 & - & 0.00 & - & - & 18 & 2.74 \\
\hline 14 & 23.73 & 132 & 19.05 & 391 & 20.30 & 27 & 2.81 & 16 & 5.73 & 657 & 100.00 \\
\hline
\end{tabular}

Table 8a. Frequency of immunization coverage among study participants $(n=4,357)$

\begin{tabular}{|c|c|c|c|c|c|c|c|}
\hline \multirow{3}{*}{ Vaccination } & \multicolumn{7}{|c|}{ Immunization Status } \\
\hline & \multicolumn{2}{|c|}{ Yes } & \multicolumn{2}{|c|}{ None } & \multicolumn{2}{|c|}{ Unknown } & \multirow{2}{*}{ Total } \\
\hline & $\mathbf{N}$ & $\%$ & $\mathbf{N}$ & $\%$ & $\mathbf{N}$ & $\%$ & \\
\hline Tetanus & 3,420 & $78.49 \%$ & 726 & $16.66 \%$ & 211 & $4.84 \%$ & 4357 \\
\hline Hepatitis B & 3,000 & $68.85 \%$ & 1,031 & $23.66 \%$ & 326 & $7.48 \%$ & 4357 \\
\hline MMR & 2,583 & $59.28 \%$ & 1,357 & $31.15 \%$ & 417 & $9.57 \%$ & 4357 \\
\hline
\end{tabular}

aside from recommending folic acid supplementation, have implemented national policies to fortify staple foods to ensure adequate supplement levels in women of childbearing age. ${ }^{18,19}$ Iron deficiency, resulting in anemia, heightens the risk for preterm birth, maternal and infant mortality, and low birthweight. ${ }^{17}$ Folic acid supplementation in the three months before conception lowers the risk for neural tube defects. ${ }^{2,17}$ In the Philippines, the Department of Health recommends the intake of a combination of $400 \mathrm{mcg}$ of Folic Acid and Iron $(60 \mathrm{mg})$ supplementation among pregnant women $^{20}$ but there is no monitoring of intake among women of reproductive age.

\section{Lifestyle check}

Only $55 \%$ of the women responders had a normal Body Mass Index (BMI). Under-nutrition was highest among women in the schools (45.58\%) and adolescents 15-19 years old (42.50\%). Interestingly, the percentage of overweight or obese women was highest among health workers (49.15\%), women 35-39 years old (44.82\%) and women 45-49 years old (45.39\%). Table 10a presents the BMI of study participants according to age and Table $10 \mathrm{~b}$ presents the BMI of study participants according to study sites. Achieving an optimal pre-pregnancy BMI reduces pregnancy risk from being underweight or obese. Infants born to underweight women are at risk for low birth weight and prematurity, while obesity results in an increased risk for preterm birth and gestational diabetes and hypertension. ${ }^{2,17}$

Nine percent (9\%) of study participants admitted alcohol use and $2.07 \%$ were smokers. Table $11 \mathrm{a}$ presents the frequency of substance abuse according to age and Table $11 \mathrm{~b}$ presents the frequency according to study site. Those who smoke and consume alcohol were advised on smoking cessation and abstinence from alcohol, as these substances can result in pregnancy loss, placental problems, low birth weight, and fetal alcohol syndrome. , $^{2,7}$

\section{Infectious Diseases}

Table $12 \mathrm{a}$ shows the presence of infectious diseases and parasitism according to age and Table $12 \mathrm{~b}$ presents data according to study site. The overall screening rate for $\mathrm{HIV}$ and other sexually transmitted infections (STIs) was $3.19 \%$ and $3.08 \%$, respectively, with concentration among entertainers for these conditions. Untreated STIs can affect a woman's fertility, resulting in pregnancy loss, preterm birth, low birth weight, congenital infections, and mother-tochild transmission of HIV., ${ }^{2,17,21}$

\section{Chronic medical conditions}

Anemia (5.16\%) and hypertension (3.17\%) topped the list of chronic medical conditions. Table $13 \mathrm{a}$ presents the occurrence of chronic medical conditions according to age of the study participants and Table $13 \mathrm{~b}$ presents data according to study sites. The diagnosis and treatment of chronic maternal diseases like diabetes, anemia, asthma, hypertension, congenital heart disease, thyroid disease, and epilepsy reduce pregnancy complications and adverse outcomes, such as prematurity and birth defects. At the same time, potentially teratogenic medication being taken, such as seizure medications and warfarin can be adjusted or replaced..$^{2,17}$ 
Table 8b. Frequency of immunization status according to age $(n=4,537)$

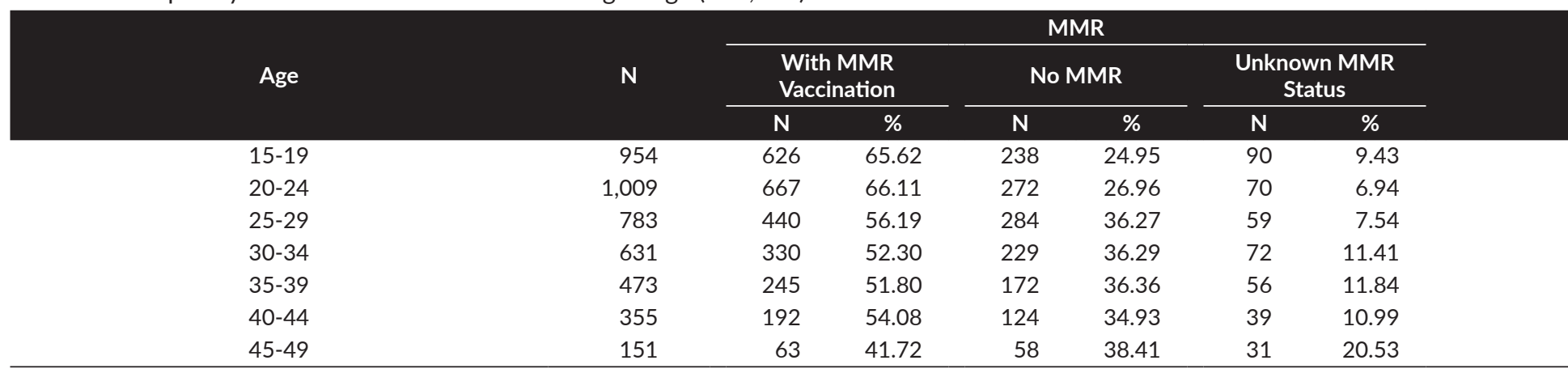

Table 8c. Frequency of immunization coverage by study site $(n=4,537)$

\begin{tabular}{|c|c|c|c|c|c|c|c|c|c|c|c|c|c|}
\hline \multirow{3}{*}{\multicolumn{4}{|c|}{ Study sites }} & \multirow{3}{*}{\multicolumn{2}{|c|}{$\mathbf{N}$}} & \multicolumn{8}{|c|}{ MMR } \\
\hline & & & & & & \multicolumn{3}{|c|}{$\begin{array}{l}\text { With MMR } \\
\text { Vaccination }\end{array}$} & \multicolumn{2}{|c|}{ No MMR } & \multicolumn{3}{|c|}{$\begin{array}{c}\text { Unknown MMR } \\
\text { Status }\end{array}$} \\
\hline & & & & & & $\mathbf{N}$ & \multicolumn{2}{|l|}{$\%$} & $\mathbf{N}$ & $\%$ & $\mathbf{N}$ & \multicolumn{2}{|r|}{$\%$} \\
\hline \multicolumn{4}{|c|}{ Industries/factories } & \multicolumn{2}{|c|}{310} & 161 & \multicolumn{2}{|c|}{51.94} & 81 & 26.13 & \multicolumn{2}{|r|}{68} & 21.94 \\
\hline \multicolumn{4}{|c|}{ City Hall/Local Government offices } & \multicolumn{2}{|c|}{84} & 57 & \multicolumn{2}{|c|}{67.86} & 21 & 25.00 & \multicolumn{2}{|r|}{6} & 7.14 \\
\hline & ffices & & & \multicolumn{2}{|c|}{24} & 9 & \multicolumn{2}{|c|}{37.5} & 15 & 62.50 & \multicolumn{2}{|r|}{-} & 0.00 \\
\hline & eachers) & & & \multicolumn{2}{|c|}{6} & 5 & \multicolumn{2}{|c|}{83.33} & - & - & \multicolumn{2}{|r|}{1} & 16.67 \\
\hline & cudents) & & & \multicolumn{2}{|c|}{962} & 693 & \multicolumn{2}{|c|}{72.04} & 167 & 17.36 & & 102 & 10.60 \\
\hline Ent & t Centers & & & 1 & & 11 & 78.57 & & 3 & 21.43 & & - & - \\
\hline & enters & & & 5 & & 31 & 52.5 & & 26 & 44.07 & & 2 & 3.39 \\
\hline & munities & & & 1,92 & & 1056 & 54.83 & & 715 & 37.12 & & 155 & 8.05 \\
\hline & munities & & & 69 & & 383 & 55.27 & & 241 & 34.78 & & 69 & 9.96 \\
\hline Pre & Seminars & & & 27 & & 117 & 41.9 & & 88 & 31.54 & & 14 & 5.02 \\
\hline 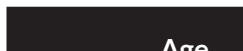 & $\mathbf{N}$ & Foli & Acid & Multiv & amins & Iron $\mathrm{T}$ & blets & Calciu & I Tablets & lodizec & Salts & & hers \\
\hline Age & $\mathbf{N}$ & $\mathbf{N}$ & $\%$ & $\mathbf{N}$ & $\%$ & $\mathbf{N}$ & $\%$ & $\mathbf{N}$ & $\%$ & $\mathbf{N}$ & $\%$ & $\mathbf{N}$ & $\%$ \\
\hline $15-19$ & 954 & 56 & 5.87 & 143 & 14.99 & 115 & 12.05 & 1 & 0.10 & 540 & 56.60 & 48 & 5.03 \\
\hline $20-24$ & 1,009 & 196 & 19.43 & 170 & 16.85 & 327 & 32.41 & 6 & 0.59 & 552 & 54.71 & 30 & 2.97 \\
\hline $25-29$ & 783 & 177 & 22.61 & 134 & 17.11 & 298 & 38.06 & 12 & 1.53 & 445 & 56.83 & 34 & 4.34 \\
\hline $30-34$ & 631 & 161 & 25.52 & 103 & 16.32 & 257 & 40.73 & 9 & 1.43 & 369 & 58.48 & 34 & 5.39 \\
\hline $35-39$ & 473 & 96 & 20.30 & 82 & 17.34 & 164 & 34.67 & 7 & 1.48 & 289 & 61.10 & 23 & 4.86 \\
\hline $40-44$ & 355 & 52 & 14.65 & 62 & 17.46 & 110 & 30.99 & 9 & 2.54 & 213 & 60.00 & 24 & 6.76 \\
\hline $45-49$ & 152 & 19 & 12.5 & 41 & 26.97 & 43 & 28.29 & 5 & 3.29 & 93 & 61.18 & 17 & 11.18 \\
\hline Total & 4,357 & 757 & 17.37 & 735 & 16.87 & 1314 & 30.16 & 49 & 1.12 & 2501 & 57.40 & 210 & 4.82 \\
\hline
\end{tabular}

Table 9b. Micronutrient intake of study participants according to study sites ( $n=4,357)$

\begin{tabular}{|c|c|c|c|c|c|c|c|c|c|c|c|c|c|}
\hline \multirow{2}{*}{ Study sites } & \multirow{2}{*}{$\mathbf{N}$} & \multicolumn{2}{|c|}{ Folic Acid } & \multicolumn{2}{|c|}{ Multivitamins } & \multicolumn{2}{|c|}{ Iron Tablets } & \multicolumn{2}{|c|}{ Calcium Tablets } & \multicolumn{2}{|c|}{ lodized Salts } & \multicolumn{2}{|c|}{ Others } \\
\hline & & $\mathbf{N}$ & $\%$ & $\mathbf{N}$ & $\%$ & $\mathbf{N}$ & $\%$ & $\mathbf{N}$ & $\%$ & $\mathbf{N}$ & $\%$ & $\mathbf{N}$ & $\%$ \\
\hline Industries/factories & 310 & 57 & 18.39 & 62 & 20.00 & 64 & 20.65 & 6 & 1.94 & 152 & 49.03 & 15 & 4.84 \\
\hline $\begin{array}{c}\text { City Hall/ } \\
\text { Local Government Offices }\end{array}$ & 84 & 29 & 34.52 & 38 & 45.24 & 36 & 42.86 & 11 & 13.10 & 58 & 69.05 & 22 & 26.19 \\
\hline Schools (Teachers) & 6 & 2 & 33.33 & 1 & 16.67 & 3 & 50.00 & - & - & 3 & 50.00 & 3 & 50.00 \\
\hline Schools (Students) & 962 & 6 & 0.62 & 145 & 15.07 & 54 & 5.61 & 2 & 0.21 & 546 & 56.76 & 56 & 5.82 \\
\hline Entertainment Centers & 14 & 6 & 42.86 & 7 & 50.00 & 7 & 50.00 & - & - & 11 & 78.57 & 1 & 7.14 \\
\hline Other Offices & 24 & 1 & 4.17 & 1 & 4.17 & 11 & 45.83 & - & - & 12 & 50.00 & 2 & 8.33 \\
\hline Health Centers & 59 & 12 & 20.34 & 18 & 30.51 & 16 & 27.12 & 6 & 10.17 & 42 & 71.19 & 14 & 23.73 \\
\hline Rural Communities & 1,926 & 383 & 19.89 & 244 & 12.67 & 720 & 37.38 & 14 & 0.73 & 1135 & 58.93 & 41 & 2.13 \\
\hline Urban Communities & 693 & 164 & 23.67 & 148 & 21.36 & 289 & 41.70 & 7 & 1.01 & 375 & 54.11 & 34 & 4.91 \\
\hline Pre-marriage seminars & 279 & 97 & 34.77 & 70 & 25.09 & 114 & 40.86 & 3 & 1.08 & 167 & 59.86 & 21 & 7.53 \\
\hline Total & 4,357 & 757 & 17.37 & 734 & 16.85 & 1,314 & 30.16 & 49 & 1.12 & 2,501 & 57.40 & 209 & 4.80 \\
\hline
\end{tabular}




\begin{tabular}{|c|c|c|c|c|c|c|c|c|c|c|c|}
\hline \multicolumn{6}{|c|}{ Tetanus } & \multicolumn{6}{|c|}{ Hepatitis B Vaccine } \\
\hline \multicolumn{2}{|c|}{$\begin{array}{l}\text { With Tetanus } \\
\text { Vaccination }\end{array}$} & \multicolumn{2}{|c|}{$\begin{array}{l}\text { No Tetanus } \\
\text { Vaccination }\end{array}$} & \multicolumn{2}{|c|}{$\begin{array}{c}\text { Unknown Tetanus } \\
\text { Status }\end{array}$} & \multicolumn{2}{|c|}{$\begin{array}{l}\text { With HEP B } \\
\text { Vaccination }\end{array}$} & \multicolumn{2}{|c|}{$\begin{array}{c}\text { No HEP B } \\
\text { Vaccination }\end{array}$} & \multicolumn{2}{|c|}{$\begin{array}{c}\text { Unknown HEP E } \\
\text { Status }\end{array}$} \\
\hline $\mathbf{N}$ & $\%$ & $\mathbf{N}$ & $\%$ & $\mathbf{N}$ & $\%$ & $\mathbf{N}$ & $\%$ & $\mathbf{N}$ & $\%$ & $\mathbf{N}$ & $\%$ \\
\hline 590 & 61.84 & 305 & 31.97 & 59 & 6.18 & 643 & 67.40 & 219 & 22.96 & 92 & 9.64 \\
\hline 770 & 76.31 & 198 & 19.62 & 41 & 4.06 & 737 & 73.04 & 217 & 21.51 & 55 & 5.45 \\
\hline 679 & 86.72 & 82 & 10.47 & 22 & 2.81 & 552 & 70.50 & 194 & 24.78 & 37 & 4.73 \\
\hline 536 & 84.94 & 67 & 10.62 & 28 & 4.44 & 430 & 68.15 & 152 & 24.09 & 49 & 7.77 \\
\hline 412 & 87.10 & 34 & 7.19 & 27 & 5.71 & 302 & 63.85 & 125 & 26.43 & 46 & 9.73 \\
\hline 314 & 88.45 & 23 & 6.48 & 18 & 5.07 & 251 & 70.70 & 76 & 21.41 & 28 & 7.89 \\
\hline 119 & 78.81 & 17 & 11.26 & 16 & 10.60 & 85 & 56.29 & 48 & 31.79 & 19 & 12.58 \\
\hline
\end{tabular}

\begin{tabular}{|c|c|c|c|c|c|c|c|c|c|c|c|}
\hline \multicolumn{6}{|c|}{ Tetanus } & \multicolumn{6}{|c|}{ Hepatitis B Vaccine } \\
\hline \multicolumn{2}{|c|}{$\begin{array}{l}\text { With Tetanus } \\
\text { Vaccination }\end{array}$} & \multicolumn{2}{|c|}{$\begin{array}{l}\text { No Tetanus } \\
\text { Vaccination }\end{array}$} & \multicolumn{2}{|c|}{$\begin{array}{c}\text { Unknown Tetanus } \\
\text { Status }\end{array}$} & \multicolumn{2}{|c|}{$\begin{array}{l}\text { With HEP B } \\
\text { Vaccination }\end{array}$} & \multicolumn{2}{|c|}{$\begin{array}{c}\text { No HEP B } \\
\text { Vaccination }\end{array}$} & \multicolumn{2}{|c|}{$\begin{array}{c}\text { Unknown HEP B } \\
\text { Status }\end{array}$} \\
\hline $\mathbf{N}$ & $\%$ & $\mathbf{N}$ & $\%$ & $\mathbf{N}$ & $\%$ & $\mathbf{N}$ & $\%$ & $\mathbf{N}$ & $\%$ & $\mathrm{~N}$ & $\%$ \\
\hline 212 & 68.39 & 112 & 36.13 & 21 & 6.77 & 182 & 58.71 & 112 & 36.13 & 42 & 13.55 \\
\hline 65 & 77.38 & 19 & 22.62 & - & - & 52 & 61.90 & 27 & 32.14 & - & - \\
\hline 23 & 95.83 & 1 & 4.17 & - & - & 11 & 45.83 & 13 & 54.17 & - & - \\
\hline 6 & 100.00 & - & 0.00 & - & - & 6 & 100.00 & - & - & - & - \\
\hline 564 & 58.63 & 325 & 33.78 & 73 & 7.59 & 662 & 68.81 & 196 & 20.37 & 104 & 10.81 \\
\hline 11 & 78.57 & 3 & 21.43 & - & - & 11 & 78.57 & 3 & 21.43 & - & - \\
\hline 58 & 98.31 & 1 & 1.69 & - & - & 49 & 83.05 & 10 & 16.95 & - & - \\
\hline 1705 & 88.53 & 151 & 7.84 & 70 & 3.63 & 1341 & 69.63 & 469 & 24.35 & 116 & 6.02 \\
\hline 559 & 80.66 & 94 & 13.56 & 40 & 5.77 & 458 & 66.09 & 186 & 26.84 & 49 & 7.07 \\
\hline 217 & 77.78 & 55 & 19.71 & 7 & 2.51 & 228 & 81.72 & 41 & 14.70 & 10 & 3.58 \\
\hline
\end{tabular}

Table 10a. Basic Metabolic Rate (BMI) of study participants according to age $(n=4,352)$

\begin{tabular}{|c|c|c|c|c|c|c|c|c|c|c|c|}
\hline \multirow{2}{*}{ Age } & \multirow{2}{*}{$\mathbf{N}$} & \multicolumn{2}{|c|}{ Underweight (<15.5) } & \multicolumn{2}{|c|}{ Normal (18.5-22.9) } & \multicolumn{2}{|c|}{ Overweight (23-24.9) } & \multicolumn{2}{|c|}{ Obese I (25-29.9) } & \multicolumn{2}{|c|}{ Obese II $(\geq 30)$} \\
\hline & & $\mathbf{N}$ & $\%$ & $\mathbf{N}$ & $\%$ & $\mathbf{N}$ & $\%$ & $\mathbf{N}$ & $\%$ & $\mathbf{N}$ & $\%$ \\
\hline $15-19$ & 953 & 405 & 42.50 & 461 & 48.37 & 52 & 5.46 & 31 & 3.25 & 4 & 0.42 \\
\hline $20-24$ & 1,007 & 299 & 29.69 & 585 & 58.09 & 67 & 6.65 & 47 & 4.67 & 9 & 0.89 \\
\hline $25-29$ & 781 & 165 & 21.13 & 449 & 57.49 & 90 & 11.52 & 60 & 7.68 & 17 & 2.18 \\
\hline $30-34$ & 631 & 99 & 15.69 & 366 & 58.00 & 79 & 12.52 & 70 & 11.09 & 17 & 2.69 \\
\hline $35-39$ & 473 & 66 & 13.95 & 259 & 54.76 & 71 & 15.01 & 64 & 13.53 & 13 & 2.75 \\
\hline $40-44$ & 355 & 63 & 17.75 & 194 & 54.65 & 55 & 15.49 & 37 & 10.42 & 6 & 1.69 \\
\hline $45-49$ & 152 & 20 & 13.16 & 80 & 52.63 & 27 & 17.76 & 17 & 11.18 & 8 & 5.26 \\
\hline Total & 4,352 & 1117 & 25.67 & 2394 & 55.01 & 441 & 10.13 & 326 & 7.49 & 74 & 1.70 \\
\hline
\end{tabular}

Table 10b. Basic Metabolic Rate (BMI) of study participants according to age $(n=4,352)$

\begin{tabular}{|c|c|c|c|c|c|c|c|c|c|c|c|}
\hline \multirow{2}{*}{ Study sites } & \multirow{2}{*}{$\mathbf{N}$} & \multicolumn{2}{|c|}{ Underweight (<15.5) } & \multicolumn{2}{|c|}{ Normal (18.5-22.9) } & \multicolumn{2}{|c|}{ Overweight (23-24.9) } & \multicolumn{2}{|c|}{ Obese I (25-29.9) } & \multicolumn{2}{|c|}{ Obese II $(\geq 30$} \\
\hline & & $\mathbf{N}$ & $\%$ & $\mathbf{N}$ & $\%$ & $\mathbf{N}$ & $\%$ & $\mathbf{N}$ & $\%$ & $\mathbf{N}$ & $\%$ \\
\hline Industries/factories & 309 & 51 & 16.50 & 184 & 59.55 & 29 & 9.39 & 31 & 10.03 & 14 & 4.53 \\
\hline $\begin{array}{c}\text { City Hall/Local } \\
\text { Government Offices }\end{array}$ & 84 & 10 & 11.90 & 45 & 53.57 & 13 & 15.48 & 12 & 14.29 & 4 & 4.76 \\
\hline Schools (Teachers) & 6 & 2 & 33.33 & 3 & 50.00 & - & - & 1 & 16.67 & - & - \\
\hline Schools (Students) & 961 & 438 & 45.58 & 459 & 47.76 & 39 & 4.06 & 24 & 2.50 & 1 & 0.10 \\
\hline Entertainment Centers & 14 & 3 & 21.43 & 11 & 78.57 & - & - & - & - & - & - \\
\hline Other Offices & 24 & 4 & 16.67 & 11 & 45.83 & 6 & 25.00 & 3 & 12.50 & - & - \\
\hline Health Centers & 59 & 3 & 5.08 & 27 & 45.76 & 13 & 22.03 & 16 & 27.12 & - & - \\
\hline Rural Communities & 1,923 & 409 & 21.27 & 1,093 & 56.84 & 222 & 11.54 & 164 & 8.53 & 35 & 1.82 \\
\hline Urban Communities & 693 & 141 & 20.35 & 398 & 57.43 & 82 & 11.83 & 60 & 8.66 & 12 & 1.73 \\
\hline Pre-marriage seminars & 279 & 56 & 20.07 & 163 & 58.42 & 37 & 13.26 & 15 & 5.38 & 8 & 2.87 \\
\hline Total & 4,352 & 1,117 & 25.67 & 2,394 & 55.01 & 441 & 10.13 & 326 & 7.49 & 74 & 1.70 \\
\hline
\end{tabular}


Table 11a. Substance use among study participants according to age $(n=4,357)$

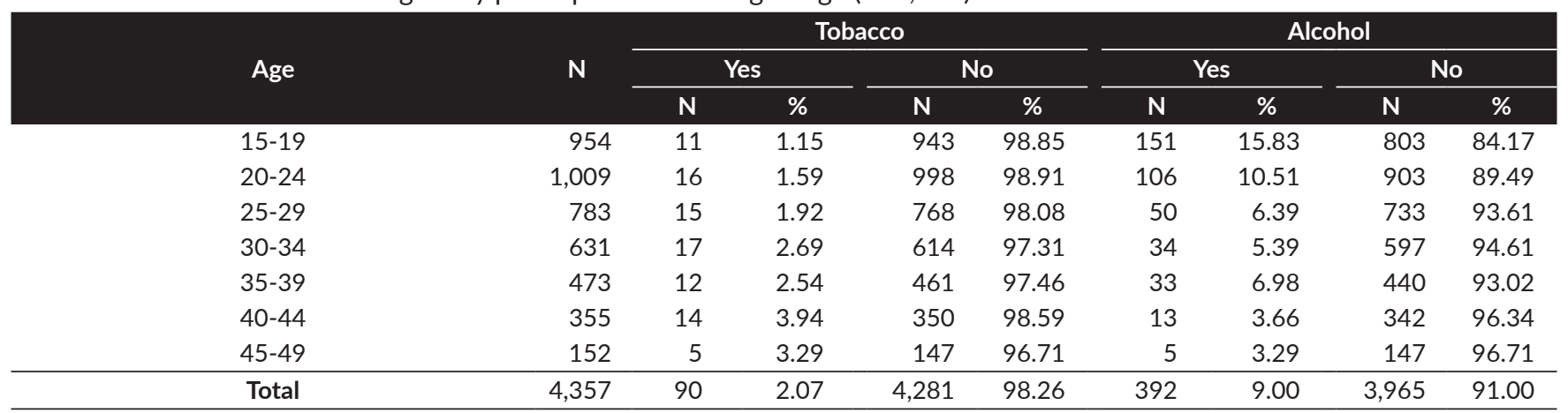

Table 11b. Substance use among study participants according to study site ( $n=4,357)$

\begin{tabular}{|c|c|c|c|c|c|c|c|c|c|}
\hline \multirow{3}{*}{ Study sites } & \multirow{3}{*}{$\mathbf{N}$} & \multicolumn{4}{|c|}{ Tobacco } & \multicolumn{4}{|c|}{ Alcohol } \\
\hline & & \multicolumn{2}{|c|}{ Yes } & \multicolumn{2}{|c|}{ No } & \multicolumn{2}{|c|}{ Yes } & \multicolumn{2}{|c|}{ No } \\
\hline & & $\mathbf{N}$ & $\%$ & $\mathbf{N}$ & $\%$ & $\mathbf{N}$ & $\%$ & $\mathbf{N}$ & $\%$ \\
\hline Industries/factories & 310 & 6 & 1.94 & 304 & 98.06 & 23 & 7.42 & 287 & 92.58 \\
\hline City Hall/Local Government offices & 84 & - & - & 84 & 100.00 & - & - & 84 & 100.00 \\
\hline Schools (Teachers) & 6 & - & - & 6 & 100.00 & - & - & 6 & 100.00 \\
\hline Schools (Students) & 962 & 10 & 1.04 & 952 & 98.96 & 162 & 16.84 & 800 & 83.16 \\
\hline Entertainment Centers & 14 & - & - & 14 & 100.00 & - & - & 14 & 100.00 \\
\hline Other Offices & 24 & - & - & 24 & 100.00 & - & - & 24 & 100.00 \\
\hline Health Centers & 59 & - & - & 59 & 100.00 & - & - & 59 & 100.00 \\
\hline Rural Communities & 1,926 & 46 & 2.39 & 1880 & 97.61 & 116 & 6.02 & 1810 & 93.98 \\
\hline Urban Communities & 693 & 26 & 3.75 & 667 & 96.25 & 81 & 11.69 & 612 & 88.31 \\
\hline Pre-marriage seminars & 279 & 2 & 0.72 & 277 & 99.28 & 10 & 3.58 & 269 & 96.42 \\
\hline Total & 4,357 & 90 & 2.07 & 4267 & 97.93 & 392 & 9.01 & 3,965 & 91.11 \\
\hline
\end{tabular}

Table 12a. Infectious diseases and parasitism among study participants according to age $(n=4,357)$

\begin{tabular}{|c|c|c|c|c|c|c|c|c|c|c|c|c|c|}
\hline \multirow{2}{*}{ Age } & \multirow[t]{2}{*}{$\mathbf{N}$} & \multicolumn{2}{|c|}{$\begin{array}{l}\text { Screened } \\
\text { for HIV }\end{array}$} & \multicolumn{2}{|c|}{ Other STI's } & \multicolumn{2}{|c|}{$\begin{array}{l}\text { Intestinal } \\
\text { Parasites }\end{array}$} & \multicolumn{2}{|c|}{$\begin{array}{l}\text { Periodontal } \\
\text { Disease }\end{array}$} & \multicolumn{2}{|c|}{$\begin{array}{c}\text { Other infectious } \\
\text { Disease }\end{array}$} & \multicolumn{2}{|c|}{$\begin{array}{c}\text { Treated for } \\
\text { Infectious Disease }\end{array}$} \\
\hline & & $\mathbf{N}$ & $\%$ & $\mathbf{N}$ & $\%$ & $\mathbf{N}$ & $\%$ & $\mathbf{N}$ & $\%$ & $\mathbf{N}$ & $\%$ & $\mathbf{N}$ & $\%$ \\
\hline $15-19$ & 954 & 8 & 0.84 & 8 & 0.84 & 165 & 17.30 & 120 & 12.58 & 4 & 0.42 & 1 & 0.10 \\
\hline $20-24$ & 1009 & 31 & 3.07 & 31 & 3.07 & 156 & 15.46 & 95 & 9.42 & 3 & 0.30 & - & - \\
\hline $25-29$ & 783 & 28 & 3.58 & 27 & 3.45 & 111 & 14.18 & 55 & 7.02 & 2 & 0.26 & - & - \\
\hline $30-34$ & 631 & 30 & 4.75 & 27 & 4.28 & 105 & 16.64 & 63 & 9.98 & 2 & 0.32 & 2 & 0.32 \\
\hline $35-39$ & 473 & 22 & 4.65 & 21 & 4.44 & 72 & 15.22 & 42 & 8.88 & 2 & 0.42 & - & - \\
\hline $40-44$ & 355 & 13 & 3.66 & 13 & 3.66 & 39 & 10.99 & 40 & 11.27 & 1 & 0.28 & 1 & 0.28 \\
\hline $45-49$ & 152 & 7 & 4.61 & 7 & 4.61 & 25 & 16.45 & 18 & 11.84 & - & - & - & - \\
\hline Total & 4,357 & 139 & 3.19 & 134 & 3.08 & 673 & 15.45 & 433 & 9.94 & 14 & 0.32 & 4 & 0.09 \\
\hline
\end{tabular}

\section{Psychological Factors}

Of the women interviewed, 20.84\% reported experiencing a stressful family life, $1.95 \%$ experienced physical violence, while $0.25 \%$ of women of reproductive age were being treated for depression. Women working in local government offices $(28.57 \%)$, teachers $(33.33 \%)$, entertainers (28.57\%), young women in schools (29.94\%), and adolescents aged 15-19 years old (26\%) were the more likely groups to experience a stressful family life. Entertainers (14.29\%) were the most likely to have experienced physical violence. Table $14 \mathrm{a}$ presents data according to age and Table $14 \mathrm{~b}$ presents data according to study site. Identifying psychosocial risk factors such as depression and domestic violence is also part of preconception care, as research shows that infants born to mothers with depression and to mothers exposed to domestic violence are at an increased risk for preterm birth and low birthweight. 1,17,22-26

\section{Focus Group Discussions}

Eleven FGDs were conducted among selected study participants. From the FGD data, thematic analyses were done and meaningful excerpts were selected. Pervasive themes were identified. There were 3 FGDs with 19 women from the community settings. There were 8 FGDs involving women in various workplaces: 8 workers from industries/factories; 6 employees from the city hall; 8 teachers and 8 students 
Table 12b. Infectious diseases and parasitism among study participants according to study site $(n=4,357)$

\begin{tabular}{|c|c|c|c|c|c|c|c|c|c|c|c|c|c|}
\hline \multirow[t]{2}{*}{ Study sites } & \multirow[t]{2}{*}{$\mathbf{N}$} & \multicolumn{2}{|c|}{$\begin{array}{l}\text { Screened } \\
\text { for HIV }\end{array}$} & \multicolumn{2}{|c|}{ Other STI's } & \multicolumn{2}{|c|}{$\begin{array}{l}\text { Intestinal } \\
\text { Parasites }\end{array}$} & \multicolumn{2}{|c|}{$\begin{array}{l}\text { Periodontal } \\
\text { Disease }\end{array}$} & \multicolumn{2}{|c|}{$\begin{array}{l}\text { Other infectious } \\
\text { Disease }\end{array}$} & \multicolumn{2}{|c|}{$\begin{array}{c}\text { Treated for } \\
\text { Infectious Disease }\end{array}$} \\
\hline & & $\mathbf{N}$ & $\%$ & $\mathbf{N}$ & $\%$ & $\mathbf{N}$ & $\%$ & $\mathbf{N}$ & $\%$ & $\mathbf{N}$ & $\%$ & $\mathbf{N}$ & $\%$ \\
\hline Industries/factories & 310 & 15 & 4.84 & 13 & 4.19 & 48 & 15.48 & 19 & 6.13 & 1 & 0.32 & - & - \\
\hline $\begin{array}{c}\text { City Hall/Local } \\
\text { Government Offices }\end{array}$ & 84 & 5 & 5.95 & 5 & 5.95 & 18 & 21.43 & 5 & 5.95 & - & - & - & - \\
\hline Schools (Teachers) & 6 & - & - & - & - & 2 & 33.33 & 1 & 16.67 & - & - & - & - \\
\hline Schools (Students) & 962 & 15 & 1.56 & 15 & 1.56 & 174 & 18.09 & 120 & 12.47 & 8 & 0.83 & - & - \\
\hline Entertainment Centers & 14 & 4 & 28.57 & 4 & 28.57 & 10 & 71.43 & 7 & 50.00 & - & - & - & - \\
\hline Other Offices & 24 & - & - & - & - & - & - & - & - & - & - & - & - \\
\hline Health Centers & 59 & 1 & 1.69 & 1 & 1.69 & 6 & 10.17 & 1 & 1.69 & - & - & - & - \\
\hline Rural Communities & 1926 & 59 & 3.06 & 59 & 3.06 & 264 & 13.71 & 186 & 9.66 & 1 & 0.05 & 4 & 0.21 \\
\hline Urban Communities & 693 & 26 & 3.75 & 23 & 3.32 & 102 & 14.72 & 73 & 10.53 & 4 & 0.58 & - & - \\
\hline Pre-marriage seminars & 279 & 14 & 5.02 & 14 & 5.02 & 49 & 17.56 & 21 & 7.53 & - & - & - & - \\
\hline Total & 4,357 & 139 & 3.20 & 134 & 3.09 & 673 & 15.52 & 433 & 9.98 & 14 & 0.32 & 4 & 0.09 \\
\hline
\end{tabular}

Table 13a. Infectious diseases and parasitism among study participants according to age $(n=4,357)$

\begin{tabular}{|c|c|c|c|c|c|c|c|c|c|c|c|c|c|c|c|c|c|}
\hline \multirow{2}{*}{ Age } & \multirow[t]{2}{*}{$\mathbf{N}$} & \multicolumn{2}{|c|}{ Diabetes } & \multicolumn{2}{|c|}{ Hypertension } & \multicolumn{2}{|c|}{ Anemia } & \multicolumn{2}{|c|}{ Hypothyroidism } & \multicolumn{2}{|c|}{ PKU } & \multicolumn{2}{|c|}{$\begin{array}{c}\text { Seizure } \\
\text { Disorder }\end{array}$} & \multicolumn{2}{|c|}{$\begin{array}{c}\text { Rheumatoid } \\
\text { Arthritis }\end{array}$} & \multicolumn{2}{|c|}{$\begin{array}{c}\text { Heart } \\
\text { Disease }\end{array}$} \\
\hline & & $\mathbf{N}$ & $\%$ & $\mathbf{N}$ & $\%$ & $\mathbf{N}$ & $\%$ & $\mathbf{N}$ & $\%$ & $\mathbf{N}$ & $\%$ & $\mathbf{N}$ & $\%$ & $\mathbf{N}$ & $\%$ & $\mathbf{N}$ & $\%$ \\
\hline $15-19$ & 954 & 4 & 0.42 & 7 & 0.73 & 35 & 3.67 & 5 & 0.52 & - & - & - & - & - & - & 5 & 0.52 \\
\hline $20-24$ & 1,009 & 2 & 0.20 & 13 & 1.29 & 56 & 5.55 & 7 & 0.69 & - & - & - & - & - & - & 3 & 0.30 \\
\hline $25-29$ & 783 & 4 & 0.51 & 25 & 3.19 & 29 & 3.70 & 8 & 1.02 & - & - & 1 & 0.13 & 3 & 0.38 & 4 & 0.51 \\
\hline $30-34$ & 631 & 5 & 0.79 & 19 & 3.01 & 39 & 6.18 & 11 & 1.74 & - & - & 1 & 0.16 & 0 & 0.00 & 1 & 0.16 \\
\hline $35-39$ & 473 & 5 & 1.06 & 26 & 5.50 & 32 & 6.77 & 12 & 2.54 & - & - & 1 & 0.21 & 8 & 1.69 & 1 & 0.21 \\
\hline $40-44$ & 355 & 8 & 2.25 & 25 & 7.04 & 22 & 6.20 & 12 & 3.38 & - & - & 0 & 0.00 & 5 & 1.41 & 5 & 1.41 \\
\hline $45-49$ & 152 & 7 & 4.61 & 22 & 14.47 & 13 & 8.55 & 4 & 2.63 & - & - & 0 & 0.00 & 14 & 9.21 & 4 & 2.63 \\
\hline Total & 4,357 & 35 & 0.80 & 137 & 3.14 & 226 & 5.19 & 59 & 1.35 & - & - & 3 & 0.07 & 30 & 0.69 & 23 & 0.53 \\
\hline
\end{tabular}

Table 13b. Chronic medical conditions of study participants according to study site $(n=4,357)$

\begin{tabular}{|c|c|c|c|c|c|c|c|c|c|c|c|c|c|c|c|c|c|}
\hline \multirow[t]{2}{*}{ Study sites } & \multirow[t]{2}{*}{$\mathbf{N}$} & \multicolumn{2}{|c|}{ Diabetes } & \multicolumn{2}{|c|}{ Hypertension } & \multicolumn{2}{|c|}{ Anemia } & \multicolumn{2}{|c|}{ Hypothyroidism } & \multicolumn{2}{|c|}{ PKU } & \multicolumn{2}{|c|}{$\begin{array}{l}\text { Seizure } \\
\text { Disorder }\end{array}$} & \multicolumn{2}{|c|}{$\begin{array}{c}\text { Rheumatoid } \\
\text { Arthritis }\end{array}$} & \multicolumn{2}{|c|}{$\begin{array}{c}\text { Heart } \\
\text { Disease }\end{array}$} \\
\hline & & $\mathbf{N}$ & $\%$ & $\mathbf{N}$ & $\%$ & $\mathbf{N}$ & $\%$ & $\mathbf{N}$ & $\%$ & $\mathbf{N}$ & $\%$ & $\mathbf{N}$ & $\%$ & $\mathbf{N}$ & $\%$ & $\mathbf{N}$ & $\%$ \\
\hline $\begin{array}{l}\text { Industries/ } \\
\text { factories }\end{array}$ & 310 & 2 & 0.65 & 12 & 3.87 & 14 & 4.52 & 5 & 1.61 & - & - & 2 & 0.65 & 2 & 0.65 & 2 & 0.65 \\
\hline $\begin{array}{l}\text { City Hall/Local } \\
\text { Government } \\
\text { offices }\end{array}$ & 84 & 3 & 3.57 & 8 & 9.52 & 7 & 8.33 & 5 & 5.95 & - & - & - & - & 5 & 5.95 & 2 & 2.38 \\
\hline Schools (Teachers) & 6 & - & - & - & - & - & - & - & - & - & - & - & - & - & - & - & - \\
\hline Schools (Students) & 962 & 4 & 0.42 & 3 & 0.31 & 49 & 5.09 & 8 & 0.83 & - & - & - & - & 1 & 0.10 & 3 & 0.31 \\
\hline $\begin{array}{l}\text { Entertainment } \\
\text { Centers }\end{array}$ & 14 & - & - & 1 & 7.14 & - & - & - & - & - & - & - & - & - & - & - & - \\
\hline Other Offices & 24 & 1 & 4.17 & - & - & - & - & - & - & - & - & - & - & - & - & - & - \\
\hline Health Centers & 59 & 4 & 6.78 & 4 & 6.78 & 1 & 1.69 & 3 & 5.08 & - & - & - & - & 1 & 1.69 & 3 & 5.08 \\
\hline $\begin{array}{c}\text { Rural } \\
\text { Communities }\end{array}$ & 1,926 & 12 & 0.62 & 66 & 3.43 & 85 & 4.41 & 21 & 1.09 & - & - & - & - & 9 & 0.47 & 6 & 0.31 \\
\hline $\begin{array}{c}\text { Urban } \\
\text { Communities }\end{array}$ & 693 & 9 & 1.30 & 38 & 5.48 & 61 & 8.80 & 16 & 2.31 & - & - & 1 & 0.14 & 9 & 1.30 & 7 & 1.01 \\
\hline $\begin{array}{l}\text { Pre-marriage } \\
\text { seminars }\end{array}$ & 279 & - & - & 6 & 2.15 & 8 & 2.87 & 1 & 0.36 & - & - & - & - & 2 & 0.72 & 2 & 0.72 \\
\hline Total & 4,357 & 35 & 0.80 & 138 & 3.17 & 225 & 5.16 & 59 & 1.35 & - & - & 3 & 0.07 & 29 & 0.67 & 25 & 0.57 \\
\hline
\end{tabular}


Table 14a. Psychosocial factors among study participants according to age $(n=4,357)$

\begin{tabular}{|c|c|c|c|c|c|c|c|}
\hline \multirow{2}{*}{ Age } & \multirow{2}{*}{$\mathbf{N}$} & \multicolumn{2}{|c|}{ Being treated for depression } & \multicolumn{2}{|c|}{ Experiencing Stressful family life } & \multicolumn{2}{|c|}{ Have experienced Physical Violence } \\
\hline & & $\mathbf{N}$ & $\%$ & $\mathbf{N}$ & $\%$ & $\mathbf{N}$ & $\%$ \\
\hline $15-19$ & 954 & 5 & 0.52 & 248 & 0.52 & 22 & 0.52 \\
\hline $20-24$ & 1,009 & 1 & 0.10 & 194 & 0.10 & 22 & 0.10 \\
\hline $25-29$ & 783 & 2 & 0.26 & 112 & 0.26 & 9 & 0.26 \\
\hline $30-34$ & 631 & 3 & 0.48 & 124 & 0.48 & 14 & 0.48 \\
\hline $35-39$ & 473 & - & - & 116 & - & 9 & - \\
\hline $40-44$ & 355 & - & - & 88 & - & 8 & - \\
\hline $45-49$ & 152 & - & - & 26 & - & 1 & - \\
\hline Total & 4,357 & 11 & 0.25 & 908 & 0.25 & 85 & 0.25 \\
\hline
\end{tabular}

Table 14b. Psychosocial factors among study participants according to study sites. ( $\mathrm{n}=4357)$

\begin{tabular}{|c|c|c|c|c|c|c|c|}
\hline \multirow{2}{*}{ Age } & \multirow{2}{*}{$\mathbf{N}$} & \multicolumn{2}{|c|}{ Being treated for depression } & \multicolumn{2}{|c|}{ Experiencing Stressful family life } & \multicolumn{2}{|c|}{ Have experienced Physical Violence } \\
\hline & & $\mathbf{N}$ & $\%$ & $\mathbf{N}$ & $\%$ & $\mathbf{N}$ & $\%$ \\
\hline Industries/factories & 310 & - & - & 27 & 8.71 & 3 & 0.97 \\
\hline $\begin{array}{l}\text { City Hall/Local } \\
\text { Government offices }\end{array}$ & 84 & - & - & 24 & 28.57 & 3 & 3.57 \\
\hline Schools (Teachers) & 6 & 1 & 16.67 & 2 & 33.33 & - & - \\
\hline Schools (Students) & 962 & 5 & 0.52 & 288 & 29.94 & 26 & 2.70 \\
\hline Entertainment Centers & 14 & - & - & 4 & 28.57 & 2 & 14.29 \\
\hline Other Offices & 24 & - & - & 4 & 16.67 & - & - \\
\hline Health Centers & 59 & - & - & 8 & 13.56 & - & - \\
\hline Rural Communities & 1,926 & 1 & 0.05 & 366 & 19.00 & 30 & 1.56 \\
\hline Urban Communities & 693 & 4 & 0.58 & 169 & 24.39 & 21 & 3.03 \\
\hline Pre-marriage seminars & 279 & - & - & 16 & 5.73 & 1 & 0.36 \\
\hline Total & 4,357 & 11 & 0.25 & 908 & 20.84 & 86 & 1.97 \\
\hline
\end{tabular}

from schools; 7 commercial sex workers; 3 physicians; 12 nurses and midwives; and 8 Barangay Health Workers from the health centers of Lipa City.

For the FGD for women of reproductive age, discussions focused on: 1) their understanding of preconception health care and its significance in pregnancy; 2) current services being offered in the community specifically for woman of reproductive age; 3) perceived preconception health care needs immediately after menarche and for women of reproductive age; 4) available preconception health care services; and 5) persons and agencies responsible for improved care of the women of reproductive age. For the FGDs for health workers, additional topics included: 1) causes of high maternal and infant mortality in the community; 2) available health services in the community to address the problems; 3) proposed additional services that can promote health among women of reproductive age; 4) role of health workers at all levels in preconception health care services; and 5) knowledge and skills needed to implement an ideal preconception health care service in the community.

Table 15 shows that there is a major gap in the services for adolescents, predominantly in medical and educational services. The gaps in medical services that were identified included the lack of free consultations, immunizations, multivitamins, and laboratory examinations. Gaps in education services included information availability at puberty and beyond. Focus on the following topics was recommended: menstrual cycle and menstrual irregularities, risk for pregnancy and signs of pregnancy, good health (adequate sleep, avoidance of stress, proper nutrition), proper hygiene, clarification on traditional beliefs and myths about menstruation, correction of misconceptions (i.e., sex education leads to sexual activity) and family planning methods. The need for active government (Department of Health and Local Government Units) participation in the implementation of the services was deemed crucial to develop the ideal set of preconception healthcare services for women of reproductive age. The Department of Education plays a major role in information dissemination with the alarming increase in teenage pregnancy in the Philippines. According to the 2017 National Demographic and Health Survey of the Philippine Statistics Authority, 9\% of Filipino women aged 15-19 have begun childbearing: $7 \%$ are already mothers and an additional $2 \%$ are pregnant with their first child. ${ }^{27}$

Table 16 presents additional challenges identified by the health workers, i.e. the recognition of promotion of a healthy lifestyle for all men and women (married and unmarried), sex education for adolescents to cover bodily changes at puberty, nutrition counseling and importance of planning a pregnancy. The need for improving knowledge and skills should not be limited to the health workers but must include parents as well as all men and women who are potential parents in the future. Education at all entry points has been 
Table 15. Themes of FGDs among women of the reproductive age

\begin{tabular}{|c|c|}
\hline Themes & Issues and Concerns raised \\
\hline $\begin{array}{l}\text { Theme } 1 \\
\text { Health services needed } \\
\text { by women immediately } \\
\text { after menarche }\end{array}$ & $\begin{array}{l}\text { Medical services } \\
\text { - Separate medical services for adolescents at the health centers } \\
\text { - Free supply of multivitamins and iron supplements at the health centers } \\
\text { Education* } \\
\text { - Information on the menstrual cycle, menstrual irregularities, dysmenorrhea, risk for pregnancy, signs of pregnancy } \\
\text { - Addressing traditional beliefs and myths about menstruation } \\
\text { - Dispelling the idea that sex education leads to sexual activity } \\
\text { - Proper hygiene } \\
\text { - Proper health and nutrition, adequate sleep, avoidance of stress } \\
\text { - Abstinence to prevent teenage pregnancy } \\
\text { *to be provided by parents, schools, barangay health workers, religious institutions, NGOs }\end{array}$ \\
\hline $\begin{array}{l}\text { Theme } 2 \\
\text { Important information } \\
\text { for a woman of } \\
\text { reproductive age }\end{array}$ & $\begin{array}{l}\text { - Likelihood of pregnancy anytime } \\
\text { - Likelihood of transmission of infection and other illnesses to baby } \\
\text { - Safe sex can avoid sexually transmitted diseases } \\
\text { - Alcohol intake and smoking can affect the baby during pregnancy } \\
\text { - Immunization before pregnancy will protect mother and baby }\end{array}$ \\
\hline $\begin{array}{l}\text { Theme } 3 \\
\text { Current services being } \\
\text { offered in the community } \\
\text { specifically for woman } \\
\text { of reproductive age }\end{array}$ & $\begin{array}{l}\text { - No known services from onset of puberty or before pregnancy (most common response) } \\
\text { - Free pap smear in health centers } \\
\text { - HPV vaccine in private clinics } \\
\text { - Medicines for STDs } \\
\text { - Occasional seminars promoting good nutrition and folic acid intake during the preconception period } \\
\text { - Wellness programs/physical fitness sponsored by local non-government organization and/or private company } \\
\text { - Pre-marriage counseling seminars for couples }\end{array}$ \\
\hline $\begin{array}{l}\text { Theme } 4 \\
\text { Perceived important } \\
\text { services in the } \\
\text { community for women } \\
\text { of reproductive age }\end{array}$ & $\begin{array}{l}\text { - Separate clinic for adolescents (should not be mixed with very young children and pregnant women) } \\
\text { - Medical services to include free supplements (ferrous sulfate, folic acid and multivitamins), vaccinations, } \\
\text { work up for women with difficulty in getting pregnant } \\
\text { - Reproductive health services (family planning methods, pap smears) } \\
\text { - Seminars on financial preparation for pregnancy/delivery and importance of insurance } \\
\text { - Seminars on menstrual disorders, sex education, family planning and spacing, prevention of unplanned } \\
\text { pregnancy, healthy pregnancy, etc. }\end{array}$ \\
\hline $\begin{array}{l}\text { Theme } 5 \\
\text { Perceived needs of a } \\
\text { woman in reproductive age } \\
\text { that will ensure a healthy } \\
\text { and safe pregnancy }\end{array}$ & $\begin{array}{l}\text { - Healthy, clean and safe pregnancy (adequate sleep, avoidance of stress, abstinence from alcohol, smoking and } \\
\text { other forms of substance abuse) } \\
\text { - Proper nutrition, proper weight } \\
\text { - Exercise programs (in public venues and workplaces), } \\
\text { - Proper hygiene }\end{array}$ \\
\hline $\begin{array}{l}\text { Theme } 6 \\
\text { Persons/agencies } \\
\text { responsible for providing } \\
\text { services for women } \\
\text { of reproductive age }\end{array}$ & $\begin{array}{l}\text { - Parents and families } \\
\text { - Government (Department of Health, Department of Social Welfare and Development, Department of Education, } \\
\text { local government units with municipal health officer, nurses, midwives, barangay health workers) as providers } \\
\text { - Youth groups, NGOs, Employers } \\
\text { - Schools }\end{array}$ \\
\hline
\end{tabular}

considered a strategy, i.e. home, school and the workplace. The health workers support the need for a separate clinic for pregnant adolescents called Teen Mom's Clinic in some hospitals in consideration of their special needs i.e. emotional/ psychosocial preparedness of motherhood, responsibilities for care of the baby while schooling, etc.

Various stakeholders and sectors have been identified to be critical in overcoming the gaps and challenges in preconception healthcare namely 1) schools: through expansion of health and science subject material to include lectures on body changes during puberty, healthy \& safe pregnancy and family planning; 2) employers (government and private): through provision of wellness lectures at the workplaces as well as provision of a safe and healthy working environment; 3) health workers (physicians, nurses, midwives, community health workers): through integration of preconception care in the management of both inpatient and outpatient care; 4) marriage counsellors: through inclusion of preconception health topics during pre-marriage counselling; 4) community social workers: through inclusion of preconception healthcare topics in seminars for out-ofschool youths. Utilization of tri-media in the delivery of messages has been highly recommended. The gender balance in all messages ensures that all parents and potential parents are covered by the recommended strategies.

To effectively institute a comprehensive preconception care on a national scale, it is imperative to have an empowered community working with both government institutions and non-government agencies. Though special mention was given to the Department of Health, Department of Interior and Local Government, Department of Education, and Department of Labor and Employment, other government agencies have a role, even as a provider of wellness programs in the workplace. 
Table 16. Themes of FGDs with health workers

\begin{tabular}{|c|c|}
\hline & Issues and Concerns raised \\
\hline $\begin{array}{l}\text { Theme } 1 \\
\text { Causes of high maternal } \\
\text { and infant mortality } \\
\text { in your community }\end{array}$ & $\begin{array}{l}\text { - Home deliveries handled specifically by traditional birth attendants or 'hilots' who cannot handle complications } \\
\text { - Insufficient number of prenatal checkups } \\
\text { - Non-attendance in Mothers' Classes where healthy pregnancy and safe delivery are discussed }\end{array}$ \\
\hline $\begin{array}{l}\text { Theme } 2 \\
\text { Health services } \\
\text { available in the } \\
\text { community to address } \\
\text { the problem }\end{array}$ & $\begin{array}{l}\text { - Prenatal care services (check-ups, vitamin supplements, tetanus toxoid vaccinations) } \\
\text { - Monthly Mothers' Classes (topics on healthy and safe pregnancy, family planning, importance of prenatal } \\
\text { and postpartum care, importance of facility delivery, importance of immunization for the babies) } \\
\text { - Visits of Barangay Health Worker } \\
\text { - Visits of Community Health Teams for tracking of pregnant mothers under Pantawid Pamilyang Pilipino Program (4P's) }\end{array}$ \\
\hline $\begin{array}{l}\text { Theme } 3 \\
\text { Proposed regular } \\
\text { services that can } \\
\text { promote health } \\
\text { among women of } \\
\text { reproductive age }\end{array}$ & $\begin{array}{l}\text { Education-related services } \\
\text { - Promotion of healthy lifestyle for all men and women (married, unmarried) } \\
\text { - Seminars for women of reproductive age (health and safe pregnancy, importance of planning a pregnancy, family } \\
\text { planning methods and nutrition counseling, livelihood) } \\
\text { - Seminars for adolescents (additional topics on bodily changes at puberty; the menstrual cycle and irregularities; } \\
\text { possibility of becoming pregnant once reaching menarche;) } \\
\text { - Engagement of employers for lectures at the workplaces } \\
\text { - Development of strategy to reach the out-of-school youth } \\
\text { - Utilization of Tri-media to deliver messages } \\
\text { Medical services } \\
\text { - Clinical and counseling services for adolescents with special attention to teenage pregnancy } \\
\text { - Preventive medical services (health promotion services for well patients, screening women with known risk factors, } \\
\text { free vaccinations i.e. tetanus, human papillomavirus, free PAP smears, free fasting blood sugar tests, etc.) } \\
\text { - Health promotion activities for healthy lifestyle i.e. exercise programs/activities in the community } \\
\text { - Free laboratory examinations - free Pap smears, free fasting blood sugar tests, free human papillomavirus } \\
\text { (HPV) vaccines } \\
\text { - Free immunizations and multivitamins } \\
\text { - Development of a center for adolescents } \\
\text { Services by government agencies } \\
\text { - Involvement of Department of Education with inclusion of additional topics on healthy pregnancy and safe delivery } \\
\text { - Involvement of the Department of Social Work and Development livelihood education training for out-of- } \\
\text { school youth } \\
\text { - Involvement of Department of Interior and Local Government since the Local Government Units are directly } \\
\text { in charge of implementing health programs } \\
\text { - Involvement of Department of Labor and Employment for programs in the workplace settings }\end{array}$ \\
\hline $\begin{array}{l}\text { Theme } 4 \\
\text { Knowledge and skills } \\
\text { needed to implement } \\
\text { an ideal preconception } \\
\text { healthcare service } \\
\text { in the community }\end{array}$ & $\begin{array}{l}\text { - Knowledge for barangay health workers (additional knowledge on treatment for simple illness of pregnant women) } \\
\text { - Skills for health workers (training on counseling, imparting better communication between parents and their } \\
\text { children, motivation of adolescents to avoid sexual activities) } \\
\text { - Knowledge for women (rights of the woman especially refusal to sexual activity) } \\
\text { - Knowledge for men (role of the man, how to take care of his partner, and respect the rights of women) } \\
\text { - Knowledge for parents (effective parenting, communication with the children) }\end{array}$ \\
\hline
\end{tabular}

Integration of preconception care into the curricula of health professionals education while aligning with the principles of transformative health professionals education could help increase awareness and application in health services. Preconception care should also be mainstreamed into all the continuing professional development activities of all health professionals.

The themes identified through the interviews and FGDs support published recommendations that interventions to reduce risks during pregnancy must be better timed before pregnancy occurs. ${ }^{2,17}$ In every encounter of a woman with her health provider, concepts of preconception care should be integrated with the aim of identifying and modifying biomedical, behavioral and social risks to a woman's health and pregnancy outcomes through prevention and management. ${ }^{2}$ Knowledge of the mother's history prepares the additional care to upcoming pregnancies showing the strong link between maternal and pediatric care. ${ }^{16,17}$ Intervention must be inter-professional and inter-sectoral because a physician alone cannot address the behavioral and social risks. While preconception care tends to concentrate on women, attention on the male partner or to both parties as a couple is also important. ${ }^{2}$ Men should be screened for risk factors, such as infertility, sexually transmitted infections, and genetic predisposition. Men can also contribute to some of the pregnancy risk factors, such as smoking and domestic violence. . $^{1728}$

The Rural Health Unit (RHU) is the basic unit for delivery of health services in the Philippines. Manned by a team composed of a physician, a nurse, midwives and barangay health workers, it delivers women's health services that include health education workshops, nutrition education, immunizations, exercise classes, adolescent health services, outreach programs, and social services. This 
is similar to the successful community health center model reported by Wilensky. ${ }^{29}$ Clinics specifically designed to deliver preconception health care have been described in the literature. ${ }^{6,30.31}$ This paper suggests additional services that can enhance the preconception health care program. The major gap of clinics specifically designed for the adolescents must be urgently pursued by both government and private hospitals in view of the increasing number of teenage pregnancies in the Philippines. ${ }^{27}$

With several component services identified to support a healthy preconception care program, it is deemed crucial that a national framework be developed by concerned government agencies to be led by the Department of Health as the highest policy-making body on health. The engagement of the private sector and other non-government agencies will be fundamental in the implementation of the framework.

\section{CONCLUSION}

The preconception health status of women of reproductive age in selected communities in the progressive city of Lipa could be a reflection of the status of women in the reproductive age in other parts of the country. Indeed, the gaps identified and recommendations generated during the interviews and FGDs will benefit the city and hopefully, be referenced by other local government units in the improvement of their health programs for women of reproductive age. The alarming increasing rate of teenage pregnancy must be given highest priority by the Department of Education with integration of safe and healthy pregnancy in the curriculum. The development of programs for men and women recognizes that parenthood is a partnership. To guarantee a successful program on preconception health care services, government must utilize an inter-sectoral and inter-disciplinary approach with the participation of various stakeholders and sectors, both government and private. The engagement of women of reproductive age in planning provides a dynamic feedback for the relevance of the planned programs.

\section{Acknowledgments}

We appreciate the logistical support given by the staff of the Lipa City Health Office. We appreciate the logistical support of the Newborn Screening Society of the Philippines, Inc. We thank Prof. Cynthia Cordero for the valuable comments on the manuscript. We also thank the following people who assisted in the project: Dr. Corazon Sabile, Khristine Joy Mojares, Michelle Mantuano, Claire Ann Ona, Nicholas Oba, Errold Gualberto, Dr. Anna Carmina Zantua, Dr. Ma-Am Joy Tumulak, Dr. Kathryn Ty, Dr. Stephanie Katalbas, and Dr. Christine Umandap.

We are grateful to the following communities who participated in the project: rural communities (Antipolo del Norte, Antipolo del Sur, Pinagkawitan, Munting Pulo,
Tipakan, and San Jose); urban communities (Bgy. Bulaknin, Bgy 1, 2,3,7 and 10); schools (Kolehiyo ng Lungsod ng Lipa, De la Salle-Lipa City, and Lipa City College); factories (Sohbi Kohgei [Phils.], Inc and Pilipinas Kyohritsu, Inc (PKI); local government offices (City Hall of Lipa City and City/Rural Health Centers).

\section{Statement of Authorship}

All authors participated in data collection and analysis, and approved the final version submitted.

\section{Author Disclosure}

All authors declared no conflicts of interest.

\section{Funding Source}

This project was funded by the March of Dimes Foundation, the Institute of Human Genetics, and the Newborn Screening Reference Center.

\section{REFERENCES}

1. Center for Disease Control and Prevention, Preconception health [Internet]. 2018 [cited 2019 Apr]. Available from: https://www.cdc. gov/preconception/overview.html.

2. Johnson K, Posner SF, Biermann J, Cordero JF, Atrash HK, Parker CS, et al. Recommendations to improve preconception health and healthcare - United States. A report of the CDC/ATSDR Preconception Care Work Group and the Select Panel on Preconception Care. MWWR Recomm Rep. 2006 Apr. 55(RR-6):1-23.

3. Reeve M, Charafeddine L, Zhong N, Padilla CD, Yunis K, Rafei RE, et al. Preconception health assessment in China, Lebanon and the Philippines: applicability to other countries. Matern Child Health J. 2014 Jul; 18(5):1066-74.

4. United Nations, United Nations millennium development goals [Internet]. 2010 [cited 2018 Jun]. Available from: https://www.un.org/ millenniumgoals/bkgd.shtml.

5. United Nations Sustainable Development Programme, Sustainable development goals [Internet]. 2019 [cited 2019 Mar]. Available from: https://www.undp.org/content/undp/en/home/sustainabledevelopment-goals.html.

6. Ebrahim J, Lo S, Zhuo J, Han J, Delvoye P, Zhu L. Models of preconception care implementation in selected countries. Matern Child Health J. 2006 Sep; 10(5 suppl):S37-42.

7. Hesketh T. Getting married in China: Pass the medical first: BMJ. 2003 Feb; 326(7383):277-9.

8. Berry RJ, Li Z, Erickson JD, Li S, Moore CA, Wang H, et al. Prevention of neural-tube defects with folic acid in China. ChinaUS Collaborative Project for Neural Tube Defect Prevention. N Engl J Med. 1999 Nov; 341(20):1485-90.

9. Department of Health, Programs [Internet]. 2018 [cited 2019 Mar]. Available from: https://www.doh.gov.ph/.

10. Department of Health, Administrative Order 08-0029: Implementing health reforms for rapid reduction of maternal and neonatal mortality [Internet]. 2008 [cited $2018 \mathrm{Mar}$ ]. Available from: http://www.doh. gov.ph.

11. National Center for Disease Prevention and Control - Department of Health, The MNCHN Manual of Operations 2011 2nd edition [Internet]. 2011 [cited 2018 Mar]. Available from: http://www.doh. gov.ph.

12. Philippine Department of Interior and Local Government, LGU profile, Lipa City, Batangas, Philippines [Internet]. 2018 [cited 2018 Jul]. Available from: http://calabarzon.dilg.gov.ph/lipacitybatangas.

13. National Statistics Office and ICF Macro, Philippines national demographic and health survey 2008. Calverton (MD) [Internet]. 
2008 [cited 2018 Feb]. Available from: http://pdf.usaid.gov/pdf_docs/ PNADR533.pdf.

14. Festin MR, Laopaiboon M, Pattanittum P, Ewens MR, HendersonSmart DJ, Crowther CA. Caesarean section in four South East Asian countries: reasons for, rates, associated care practices and health outcomes. BMC Pregnancy Childbirth. 2009 May; 9:17.

15. Juarez F, Cabigon J, Singh S, Hussain R. The incidence of induced abortion in the Philippines: Current level and recent trends. Int Fam Plan Perspect. 2005 Sep; 31(3):140-9.

16. Dolan SM, Moore C. Linking family history in obstetric and pediatric care: Assessing risk for genetic disease and birth defects. Pediatrics. 2007; 120(Supp12):S66-70.

17. Dean S, Bhutta Z, Mason EM, Howson CP. Chapter 3: Care before and between pregnancy. In: Born Too Soon: The global action report on preterm birth. World Health Organization. 2012.

18. Castilla EE, Orioli IM, Lopez-Camelo JS, Dutra M da G, NazerHerrera J, Latin America Collaborative Study of Congenital Malformations (ECLMAC). Preliminary data on changes in neural tube defect prevalence rates after folic acid fortification in South America. Am J Med Genet A. 2003 Dec; 123A(2):123-8.

19. Honein M, Paulozzi L, Mathews T, Erickson J, Wong L. Impact of folic acid fortification of the US food supply on the occurrence of neural tube defects. JAMA. 2001 Jun; 285(23):2981-6.

20. MS-MOP Technical Working Group. Micronutrient Supplementation (MS) Manual of Operations (MOP). Manila: Department of Health; 2010.p 55-58.

21. Mullick S, Watson-Jones D, Beksinska M, Mabey D. Sexually transmitted infections in pregnancy: Prevalence, impact on pregnancy outcomes, and approach to treatment in developing countries. Sex Transm Infect. 2005 Aug; 81(4):294-302.

22. Shah PS, Shah J. Maternal exposure to domestic violence and pregnancy and birth outcomes: A systematic review and meta-analyses. J Women's Health. 2010 Nov; 19(11):2017-31.
23. Sarkar NN. The impact of intimate partner violence on women's reproductive health and pregnancy outcome. J Obstet Gynaecol. 2008 Apr; 28(3):266-71.

24. Yonkers KA, Wisner KL, Stewart DE, Oberlander TF, Dell DL, Stotland N, et al. The management of depression during pregnancy: A report from the American Psychiatric Association and the American College of Obstetricians and Gynecologists. Gen Hosp Psychiatry. 2009 Sep-Oct; 31(5):403-13.

25. Grote N, Bridge J, Gavin A. A meta-analysis of depression during pregnancy and the risk of pre-term birth, low birth weight, and intrauterine growth restriction. Arch Gen Psychiatry. 2010 Oct; 67(10):1012-24.

26. World Health Organization, Violence against women [Internet]. 2017 [cited $2019 \mathrm{Mar}$. Available from: https://www.who.int/news-room/ fact-sheets/detail/violence-against-women

27. 2017 National Demographic and Health Survey [Internet]. [cited 2020 Jan]. Available from: https://www.dhsprogram.com/pubs/pdf/ SR253/SR253.pdf.

28. Warner JN, Frey KA. The well-man visit: Addressing a man's health to optimize pregnancy outcomes. J Am Board Fam Med. 2013 MarApr; 26(2):196-202.

29. Wilensky S, Proser M. Community approaches to women's health: Delivering preconception care in a community health center model. Women's Health Issues. 2008 Nov-Dec; 18(6 Suppl):S52-60.

30. Boulet SL, Parker C, Atrash H. Preconception care in international settings. Matern Child Health J. 2006 Sep; 10(5 Suppl):S29-35.

31. Chamberlain G. The prepregnancy clinic. Br Med J. 1980 Jul; 281(6232):29-30. 